\title{
CONVERGENCE OF GENERALIZED COLLATZ PROBLEM IN $k$-ADIC FIELD
}

\section{YUSHU ZHU, SENSEN CHEN and QING-YOU SUN}

Hangzhou Normal University

Hangzhou 311121

P. R. China

e-mail: qysun@hznu.edu.cn

\begin{abstract}
In this article, we define a new $k$-adic series transformation called $\mathcal{Z}$ transformation and probe into its fixed point and periodicity. We extend the number field of the transform period problem to a wider $k$-adic field. Different constraints are imposed on $k$, then different periodic columns are formed after finite $\mathcal{Z}$ transformations. We obtain that their periodic sequences are $M_{1}=\{1,2\}$ and $M_{2}=\{1,2\} \bigcup\left\{n_{0}\right\} \bigcup\left\{n^{\prime}\right\}$ respectively after derivation. As an application, it can provide a reference for $\mathrm{C}$ problems in more complex algebraic systems.
\end{abstract}

2020 Mathematics Subject Classification: 11B83, 11H06, 11B37.

Keywords and phrases: $k$-adic, Collatz problem, $\mathcal{Z}$-transformation, mathematical induction, fixed point.

Received September 12, 2020

$$
\text { (C) } 2020 \text { Scientific Advances Publishers }
$$

This work is licensed under the Creative Commons Attribution International License (CC BY 3.0).

http://creativecommons.org/licenses/by/3.0/deed.en_US

Open Access (cc) (1)




\section{Introduction}

The Collatz conjecture is named after Lothar Collatz, who introduced the idea in 1937. It has been widely studied in the past 100 years, and many achievements with great value have been obtained, although the Collatz problem cannot be effectively solved.

The results of the Collatz problem can be applied to modern cryptography, and can be easily transformed into graph theory, and then extended to a wider range of algebraic and algebraic geometry. The essence of the Collatz problem is a fixed-point problem, its research methods and results are of great significance to the study of fixed point problems, and thus have an important impact on the development of modern mathematics. The problem also has important applications in power systems and fractal geometries.

The Collatz problem is concerned with the function $f: \mathbb{Z}^{+} \rightarrow \mathbb{Z}^{+}$ defined as [1]

$$
f(n)=\left\{\begin{array}{c}
\frac{n}{2}, \text { when } n \text { is even, } \\
\frac{3 n+1}{2}, \text { when } n \text { is odd. }
\end{array}\right.
$$

The famous Collatz problem asserts that iteration series $f(n), f^{2}(n)$, $f^{3}(n), \cdots$ of every positive number $n$ will eventually reach the integer one. This conjecture is still an unsolved mystery, many articles have studied this issue.

Matthews and Watts [2] gave generalized Collatz maps

$$
f_{d, m, r}(x)=\frac{m_{i} x+r_{i}}{d}, \text { if } x \equiv i(\bmod d)
$$

where $d>1$ is an integer, $m$ and $r$ are $d$-dimensional vectors such that $m_{i} x+r_{i} \equiv 0(\bmod d)$ for all $i$. The number $n$ is supposed to be cyclic for

$f_{d, m, r}$ if the series $\left\{f_{d, m, r}^{i}(n)\right\}_{i \in \mathbb{N}}$ is a periodic column. As is known, we can see that the density of the set of the integer will stop for 1 after finite times of Collatz maps which lead us to seek for other periodic column. 
Certainly, these problems and their promotion problems have many research results, especially through computers to solve these problems ([3]-[6]). The results of this article are mainly inspired by [7] and [8], which extend the problem to $p$-adic. This led us to consider this problem from a deeper perspective of domain theory, which makes us want to study a class of number theory problem.

In Sections 2 and 3, we introduce some notations and definitions, and give our main results. In Section 4, by studying the properties of the $\mathcal{Z}$-transform, Lemma 2 mentions that when the digit of $n_{0}$ is greater than 2 , then after finite $\mathcal{Z}$-transformations, it can degenerate into a single-digit number or a double-digit number in $k$-adic. So, we mainly discuss these two cases in the proof of Theorem 1. For both cases, we discuss the result of $n_{0}$ after finite $\mathcal{Z}$-transformations. Using the mathematical induction method, we obtain that after finite $\mathcal{Z}$-transformation under the hypothesis, there is only one periodic column $M_{1}=\{1,2\}$. In Theorem 2, we weaken the restriction on $k$, then proved that the period column of the $\mathcal{Z}$-transformation is listed as $M_{2}=\{1,2\} \cup\left\{n_{0}\right\} \cup\left\{n^{\prime}\right\}$.

\section{Notation and Definition}

For any given $m$-digit integer expressed in $k$-adic

$$
n_{0}=a_{m-1} k^{m-1}+a_{m-2} k^{m-2}+\cdots+a_{1} k+a_{0},
$$

where $a_{i} \in \mathbb{Z}_{0}^{+}, 0 \leq a_{i}<k(0 \leq i \leq m-1)$, and $a_{m-1} \neq 0$. 
We construct the function in $k$-adic,

$$
f(n)=\left\{\begin{array}{cc}
\frac{(n+p-1)(n+2 p-1)}{p \cdot p} & n \equiv 1(\bmod p), \\
\frac{n+p-2}{p} & n \equiv 2(\bmod p), \\
\vdots & n=p-1(\bmod p), \\
\frac{n+1}{p} & n \equiv 0(\bmod p), \\
\frac{n}{p} &
\end{array}\right.
$$

where $0 \leq n<k$.

Definition 1. Let $\mathcal{Z}\left(n_{0}\right)=\sum_{i=0}^{m-1} f\left(a_{i}\right)$, where $n_{0}, a_{i}$ and $f$ are defined as above, we call it $\mathcal{Z}$-transformation of $n_{0}$ in $k$-adic.

Definition 2. Transformation sequence $\left\{\mathcal{Z}^{i}\left(n_{0}\right)\right\},\left(i \in \mathbb{Z}_{0}^{+}\right)$, is called $\mathcal{Z}$-transformation sequence in $k$-adic.

Denote $n_{i}=\mathcal{Z}^{i}\left(n_{0}\right)$, in which $\mathcal{Z}^{0}\left(n_{0}\right)=n_{0}$, that is, $\left\{n_{i}\right\},\left(i \in \mathbb{Z}_{0}^{+}\right)$, is also the $\mathcal{Z}$-transformation sequence. It's clear that $n_{i} \in \mathbb{N}$.

Our article mainly discusses the periodic column of $\mathcal{Z}$-transform sequence.

\section{The Main Results}

\section{Hypothesis:}

(a) $2 p-1 \leq k \leq 3 p^{2}$, when $p \geq 3$;

(b) For any nonnegative integer $q$ less than $\sqrt{k}$, it satisfies $(q+1)$ $(q+2) \not \equiv-1(\bmod p)$. 
(c) For any nonnegative integer $q$ less than $\sqrt{k}$, it satisfies $(q+1)$ $(q+2) \equiv k(\bmod p)$, and $k \neq(q+1)(q+2)-1-q p$.

Under the Hypothesis, we give our main result as the following two theorems.

Theorem 1. If the Hypothesis is established, then after finite $\mathcal{Z}$-transformations, $\mathcal{Z}$-transformed sequence $\left\{n_{i}\right\}, i \in \mathbb{N}$ only has one period column $M_{1}=\{1,2\}$.

Theorem 2. If only (a) in the Hypothesis is hold, then after finite $\mathcal{Z}$-transformations, $\mathcal{Z}$-transformed sequence $\left\{n_{i}\right\}, i \in \mathbb{N}$ has the period column $M_{2}=\{1,2\} \bigcup\left\{n_{0}\right\} \cup\left\{n^{\prime}\right\}$, where $n^{\prime}$ is an integer which satisfies $\mathcal{Z}\left(n^{\prime}\right)=n^{\prime}$.

\section{The Proof of the Theorems}

To prove the theorems, we give two lemmas first.

Lemma 1. The result of adding two k-adic numbers is the same as the result of adding them in decimal to become $k$-adic.

This conclusion is obvious (see [2]). We omit it.

Lemma 2. For any given $m$-digit positive integer $n_{0}$ in $k$-adic, under the Hypothesis, $\mathcal{Z}\left(n_{0}\right)$ is an integer not exceeding $(m-1)$-digit in $k$-adic when $m \geq 3$.

Proof. Denote

$$
k=t p+s+1
$$

where $r, s \in \mathbb{N}$ and $1 \leq s \leq p$.

Due to $0 \leq a_{i} \leq k-1$, by the definition of $f(n)$ in (3), it obtains

$$
f\left(a_{i}\right) \leq f(t p+1)=(t+1)(t+2) .
$$


Thus, $\mathcal{Z}\left(n_{0}\right) \leq m(t+1)(t+2)$.

For $m=3$, by (a) in the Hypothesis, we have

$$
\mathcal{Z}\left(n_{0}\right) \leq 3(t+1)(t+2)<k^{3-1} .
$$

For $m=j$, we assume that it holds

$$
\mathcal{Z}\left(n_{0}\right) \leq j(t+1)(t+2)<k^{j-1}
$$

Then, for $m=j+1$, it's obviously that

$$
\mathcal{Z}\left(n_{0}\right) \leq(j+1)(t+1)(t+2)<k^{j-1} \cdot \frac{j+1}{j}<k^{(j+1)-1} .
$$

Therefore, by mathematical induction, we have

$$
\mathcal{Z}\left(n_{0}\right)<k^{m-1}
$$

where $m \geq 3$. That means, $\mathcal{Z}\left(n_{0}\right)$ is an integer not exceeding $(m-1)$-digit in $k$-adic when $m \geq 3$. Thus, the proof of Lemma 2 is done.

Proof of Theorem 1. For any given $m$-digit positive integer $n_{0}$ in $k$-adic, we divide the proof of Theorem 1 into three parts. Here, we can omit the case $n_{0}=1$, which make Theorem 1 be true obviously.

Case 1. $n_{0}$ is a single-digit number.

In this case, it is easy to show that $1<n_{0}<k$, and $\mathcal{Z}\left(n_{0}\right)=f\left(n_{0}\right)$.

(I) If $n_{0} \neq \equiv 1(\bmod p)$, by the definition of $f(n)$, we can easily get $\mathcal{Z}\left(n_{0}\right)=f\left(n_{0}\right)<n_{0}$. It implies that the $\mathcal{Z}$ transformation make $\mathcal{Z}\left(n_{0}\right)$ smaller than $n_{0}$, and $\mathcal{Z}\left(n_{0}\right)$ is still a single-digit number. Therefore, there exists a non-negative integer $\lambda$, which satisfies $\mathcal{Z}^{\lambda}\left(n_{0}\right)=1$, or $\mathcal{Z}^{\lambda}\left(n_{0}\right) \equiv 1(\bmod p)$. The second case is just what we will discuss next. 
Analogously, in the rest of the proof, we just need to prove that there exists a finite integer $\lambda$ make $\mathcal{Z}^{\lambda}\left(n_{0}\right)<n_{0}$. Since we can think $\mathcal{Z}^{\lambda}\left(n_{0}\right)$ as a new $n_{0}$, and do the same discussion again and again, until $\mathcal{Z}^{\lambda}\left(n_{0}\right)=1$.

(II) If $n_{0} \equiv 1(\bmod p)$ and $n_{0} \neq 1$, let $n_{0}=r p+1$. So, it can obtain that

$$
\mathcal{Z}\left(n_{0}\right)=f\left(n_{0}\right)=(r+1)(r+2)
$$

Assume that $\mathcal{Z}\left(n_{0}\right)=a_{1} k+b_{1}$, where $a_{1}, b_{1} \in \mathbb{Z}_{0}^{+}$and $b_{1}<k$. Using (a) in the Hypothesis and the definition of $f$, it can be obtained by direct calculation that $a_{1} \leq 3$.

(i) When $a_{1}=0$, that is $\mathcal{Z}\left(n_{0}\right)=f\left(n_{0}\right)=(r+1)(r+2)<k$.

If $(r+1)(r+2) \neq \equiv 1(\bmod p)$, we deduce that

$$
\mathcal{Z}^{2}\left(n_{0}\right)=\mathcal{Z}((r+1)(r+2))=\frac{(r+1)(r+2)+\theta}{p}
$$

where $\theta$ is a nonnegative integer less than $p$. Noticing $n_{0}>1$ which we discussing here, then by direct calculation, $\mathcal{Z}^{2}\left(n_{0}\right) \geq n_{0}$ is only available when $n_{0}>p\left(p^{2}-3\right)$. On another hand, $f\left(n_{0}\right)<k$ implies $n_{0}<p(p-3)$ $+1<p\left(p^{2}-3\right)$ when $p>7$. Thus, it indicates that $\mathcal{Z}^{2}\left(n_{0}\right)<n_{0}$.

If $(r+1)(r+2) \equiv 1(\bmod p)$, and $\mathcal{Z}\left(n_{0}\right)>n_{0}$ are established, then for the finite number $k$, there exists $\lambda$ such that $\mathcal{Z}^{\lambda}\left(n_{0}\right)>k$, we will discuss it in (ii) and (iii). What we want to explain here is that $\mathcal{Z}^{\lambda}\left(n_{0}\right)>$ $\mathcal{Z}^{\lambda-1}\left(n_{0}\right)$ in this case. Also, for the integer $n_{0}, \mathcal{Z}\left(n_{0}\right)=n_{0}$ does not hold. 
(ii) When $a_{1}=1$ that is $\mathcal{Z}\left(n_{0}\right)=k+b_{1}$.

If $b_{1} \not \equiv 1(\bmod p)$, we have

$$
\mathcal{Z}^{2}\left(n_{0}\right)=f(1)+f\left(b_{1}\right)=2+\frac{b_{1}+\theta}{p}<\frac{k}{p}+3
$$

where $\theta$ is a nonnegative integer less than $p$. Noting (a) in the Hypothesis, it follows that

$$
\mathcal{Z}^{2}\left(n_{0}\right)<\frac{k}{p}+3<k \leq n_{0}
$$

If $b_{1} \equiv 1(\bmod p)$, then

$$
\mathcal{Z}^{2}\left(n_{0}\right)=f(1)+f\left(b_{1}\right)=2+f\left(b_{1}\right) \triangleq a_{2} k+b_{2}
$$

For $\mathcal{Z}^{i}\left(n_{0}\right), i>2$, we can make the similar notation. And we only discuss the case $b_{i} \equiv 1(\bmod p)$, otherwise, it will be the case we discussed above. Denote $b_{i}=q_{i} p+1 . b_{i}<k$ means $q_{i}<\frac{k}{p}$. Similar to the discussion about $a_{1}$, it can be obtained by direct calculation that $a_{2} \leq 3$.

(A) Suppose that $\mathcal{Z}^{2}\left(n_{0}\right)=b_{2}<k$ is established, we only need to deal with the case $\mathcal{Z}^{2}\left(n_{0}\right) \geq n_{0}$. If $\mathcal{Z}^{2}\left(n_{0}\right)>n_{0}$, then by the definition of $f(n)$, for the finite number $k$, there exists $\lambda$ such that $\mathcal{Z}^{\lambda}\left(n_{0}\right)>k$, we will discuss it in the following. On the other hand, we have $\mathcal{Z}^{2}\left(n_{0}\right) \neq n_{0}$ when $q_{1}$ satisfies (b) in the Hypothesis, since $\mathcal{Z}^{2}\left(n_{0}\right)=f\left(b_{1}\right)+2=\left(q_{1}+1\right)$ $\left(q_{1}+2\right)+2 \neq r p+1$. 
(B) Suppose that $\mathcal{Z}^{2}\left(n_{0}\right)=k+b_{2}$ is established, then we have $b_{1} \neq b_{2}$. Otherwise, $f\left(b_{1}\right)+2=\mathcal{Z}^{2}\left(n_{0}\right)=\mathcal{Z}\left(n_{0}\right)=f\left(n_{0}\right)$, that is $\left(q_{1}+1\right)$ $\left(q_{1}+2\right)+2=(r+1)(r+2)$. This can not be established for any nonnegative integers $r$ and $q_{1}$.

If $b_{2}<b_{1}$, then $f\left(b_{2}\right)<f\left(b_{1}\right)$, that will make $\mathcal{Z}^{i}\left(n_{0}\right)<\mathcal{Z}^{i-1}\left(n_{0}\right)$, until $\mathcal{Z}^{i}\left(n_{0}\right)<k$. Also, if $q_{i-1}$ satisfies (b) in the Hypothesis, it's easy to get $\mathcal{Z}^{i}\left(n_{0}\right) \neq n_{0}$. Then, there are finite $i$ which satisfy $n_{0}<\mathcal{Z}^{i}\left(n_{0}\right)<k$ and different from each other. Thus, there exists $\lambda$ such that $\mathcal{Z}^{\lambda}\left(n_{0}\right)<n_{0}$ or $b_{\lambda+1}>b_{\lambda}$.

If $b_{2}>b_{1}$, that will make $\mathcal{Z}^{i}\left(n_{0}\right)>\mathcal{Z}^{i-1}\left(n_{0}\right)$, until $\mathcal{Z}^{i}\left(n_{0}\right) \geq 2 k$. We will discuss it next.

(C) Suppose that $\mathcal{Z}^{2}\left(n_{0}\right)=2 k+b_{2}$ is established.

If $b_{2}<b_{1}$, then $\mathcal{Z}^{3}\left(n_{0}\right)=1+f\left(b_{2}\right)<2+f\left(b_{1}\right)=\mathcal{Z}^{2}\left(n_{0}\right)$. Thus, there exists $i \geq 2$ such that $\mathcal{Z}^{i}\left(n_{0}\right)=k+b_{i}$ or $\mathcal{Z}^{i}\left(n_{0}\right)<k$, which we have discussed in (A) and (B). The only difference is the way to prove $\mathcal{Z}^{i}\left(n_{0}\right) \neq n_{0}$. Assume $\mathcal{Z}^{i}\left(n_{0}\right)=f(2)+f\left(b_{i-1}\right)=1+\left(q_{i-1}+1\right)\left(q_{i-1}+2\right)=n_{0}$, we deduce $\left(q_{i-1}+1\right)\left(q_{i-1}+2\right) \equiv 0(\bmod p)$ since $n_{0} \equiv 1(\bmod p)$. It means $q_{i-1}+1=p$, or $q_{i-1}+2=p$ when $q_{i-1}<\sqrt{k}$. It implies $n_{0}=p(p+1)+1$, or $n_{0}=(p-1) p+1$. That is, $\mathcal{Z}\left(n_{0}\right)=(p+2)(p+3)$, or $\mathcal{Z}\left(n_{0}\right)=p$ $(p+1)$. Noting $n_{0}<k<\mathcal{Z}\left(n_{0}\right)$, from the first one we have $\mathcal{Z}\left(n_{0}\right)=$ $(p(p+1)+2)+(4 p+4)$, then $\mathcal{Z}^{2}\left(n_{0}\right) \leq f(1)+f(4 p+1)=21 \leq 2(p(p+1)$ $+2) \leq 2 k$ when $p \geq 3$. And from the second one we have $\mathcal{Z}\left(n_{0}\right)=$ $(p(p-1)+2)+(2 p-2), \quad$ then $\quad \mathcal{Z}^{2}\left(n_{0}\right) \leq f(1)+f(p+1)=8 \leq p(p-1)$ $+2 \leq k$ when $p \geq 3$. These two case both contradict $\mathcal{Z}^{2}\left(n_{0}\right)=2 k+b_{2}>2 k$. 
If $b_{2}=b_{1}$, then $\left(2+f\left(b_{1}\right)\right)-\left(1+f\left(b_{2}\right)\right)=1$, so $\quad \mathcal{Z}^{3}\left(n_{0}\right) \neq a_{3} k+b_{3}$ when $\mathcal{Z}^{2}\left(n_{0}\right)=a_{2} k+b_{2}$, where $b_{i}=q_{i} p+1$. That will be the case $b_{i} \equiv 1(\bmod p)$.

If $b_{2}>b_{1}$, that will make $\mathcal{Z}^{i}\left(n_{0}\right)>\mathcal{Z}^{i-1}\left(n_{0}\right)$, until $\mathcal{Z}^{i}\left(n_{0}\right) \geq 3 k$. We will discuss it next.

(D) Suppose that $\mathcal{Z}^{2}\left(n_{0}\right)=3 k+b_{2}$ is established, this will be similar to $a_{1}=3$. We will discuss it in (iv).

(iii) When $a_{1}=2$, that is $\mathcal{Z}\left(n_{0}\right)=2 k+b_{1}$, which deduces $\mathcal{Z}^{2}\left(n_{0}\right)=$ $1+f\left(b_{1}\right)$. Then we can make a similar discussion as what we do in (ii).

It should be noted that, in this case we need the condition $\left(q_{i}+1\right)$ $\left(q_{i}+2\right) \neq \equiv(\bmod p)$, that is the first condition of (c) in the Hypothesis, to obtain $b_{1} \neq b_{2}$ corresponding to what we did in (B) of (ii).

(iv) When $a_{1}=3$, that is $\mathcal{Z}\left(n_{0}\right)=3 k+b_{1}$.

Since $n_{0}=r p+1$ and $n_{0}<k$, then by solving $\mathcal{Z}\left(n_{0}\right)=(r+1)(r+2)$ $\geq 3 k \geq 3 r p+6$, it implies that $n_{0}=3 p^{2}-p+1$ or $n_{0}=3 p^{2}-2 p+1$.

If $n_{0}=3 p^{2}-p+1$, then $\mathcal{Z}\left(n_{0}\right)=f\left(n_{0}\right)=3 p(3 p+1)=3 k+b_{1}$, and $k \geq 3 p^{2}-p+2$. This indicates $b_{1} \leq 6 p-6$. Noting $p \geq 3$, when $p \geq 4$, $\mathcal{Z}^{2}\left(n_{0}\right)=f(3)+f\left(b_{1}\right) \leq 43<n_{0}$. And when $p=3, \mathcal{Z}^{2}\left(n_{0}\right)=f(3)+f\left(b_{1}\right)$ $\leq 23<n_{0}$.

If $n_{0}=3 p^{2}-p+1$, we can get the similar conclusion by the same direct calculation.

In summary, we have a brief proof of Theorem 1 for Case 1. 
Case 2. $n$ is a 2-digit number.

Denote $n_{0}=c_{0} k+d_{0}$, then $\mathcal{Z}\left(n_{0}\right)=f\left(c_{0}\right)+f\left(d_{0}\right) \triangleq c_{1} k+d_{1}$. By what we discuss in (II) of Case 1 , since $c_{0}, d_{0}<k$, it will imply $f\left(c_{0}\right), f\left(d_{0}\right) \leq$ $3 k+(6 p-6)$. Thus, $\mathcal{Z}\left(n_{0}\right)=c_{1} k+d_{1}<7 k+d_{1}$, then, $\mathcal{Z}^{2}\left(n_{0}\right)=f\left(c_{1}\right)+$ $f\left(d_{1}\right) \leq 2+f\left(d_{1}\right)<3 k+(6 p-4)$.

If $d_{1} \neq \equiv 1(\bmod p)$, the conclusion is clearly right.

If $d_{1} \equiv 1(\bmod p)$, think $2+f\left(d_{1}\right)$ as $a_{1} k+b_{1}$ in (II) of Case 1 , then by the same way we did, there exists a nonnegative integer $\lambda$, which satisfies $\mathcal{Z}^{\lambda}\left(\mathcal{Z}\left(n_{0}\right)\right)=1$. In this case, we will use (c) in the Hypothesis to make sure that $\mathcal{Z}^{\lambda}\left(\mathcal{Z}\left(n_{0}\right)\right) \neq n_{0}$.

Thus, we finish the proof of Theorem 1 for Case 2 .

Case 3. $n$ is an $m$-digit number, where $m \geq 3$.

In this case, by Lemma 2, we can easily get that there exists a nonnegative integer $\lambda, \lambda \leq m-2$, which satisfies $\mathcal{Z}_{k}^{\lambda}(n)$ is a 2 -digit number. It will become Case 2.

Overall, it is not difficult to see that there will exist a nonnegative integer $\lambda$, which satisfies $\mathcal{Z}^{\lambda}(n)=1$. And noting $\mathcal{Z}(1)=f(1)=2, \mathcal{Z}(2)=$ $f(2)=1$, therefore, for any positive integer $\mu$, which satisfies $\mu \geq \lambda$, we have $\mathcal{Z}^{\mu}(n) \in\{1,2\}$.

Hence, Theorem 1 is proved. 
Proof of Theorem 2. The proof of Theorem 2 is similar to the proof of Theorem 1. The only difference is that, without (c) in the Hypothesis, we can not obtain $\mathcal{Z}\left(n_{0}\right) \neq n_{0}$ and $\mathcal{Z}^{i}\left(b_{i}\right) \neq \mathcal{Z}^{i-1}\left(b_{i}\right)$. Therefore, we need to add $\left\{n_{0}\right\} \cup\left\{n^{\prime}\right\}$ to the period column, where $n^{\prime}$ is an integer which satisfies $\mathcal{Z}\left(n^{\prime}\right)=n^{\prime}$.

Hence, Theorem 2 is proved.

\section{Examples}

In this section, we give some examples for different $n, k$ and $p$, to see the periodic characteristic of the $\mathcal{Z}$ transformation.

Example 1. Take $n=123789, k=137$, and $p=11$, this set satisfies the requirement of the Hypothesis, that is Theorem 1.

We can see from Figure 1 that by three times of the $\mathcal{Z}$ transformation, $n=123789$ will become 1 in 137 -adic.

Example 2. Take $n=9827, k=5$, and $p=3$, this set satisfies (a) in the Hypothesis. But when $q=0<\sqrt{5}$, it doesn't satisfy (b).

Example 3. Take $n=8512, k=10$, and $p=5$, this set also satisfies (a) in the Hypothesis. But when $q=3<\sqrt{10}$, the first requirement in (c) is not satisfied.

Examples 2 and 3 are still satisfies the requirement of Theorem 2 . From Figure 2, we can see that $\left\{n^{\prime}\right\}$ in Theorem 2 for them are $\{4,6\}$ and $\{6\}$, respectively. 


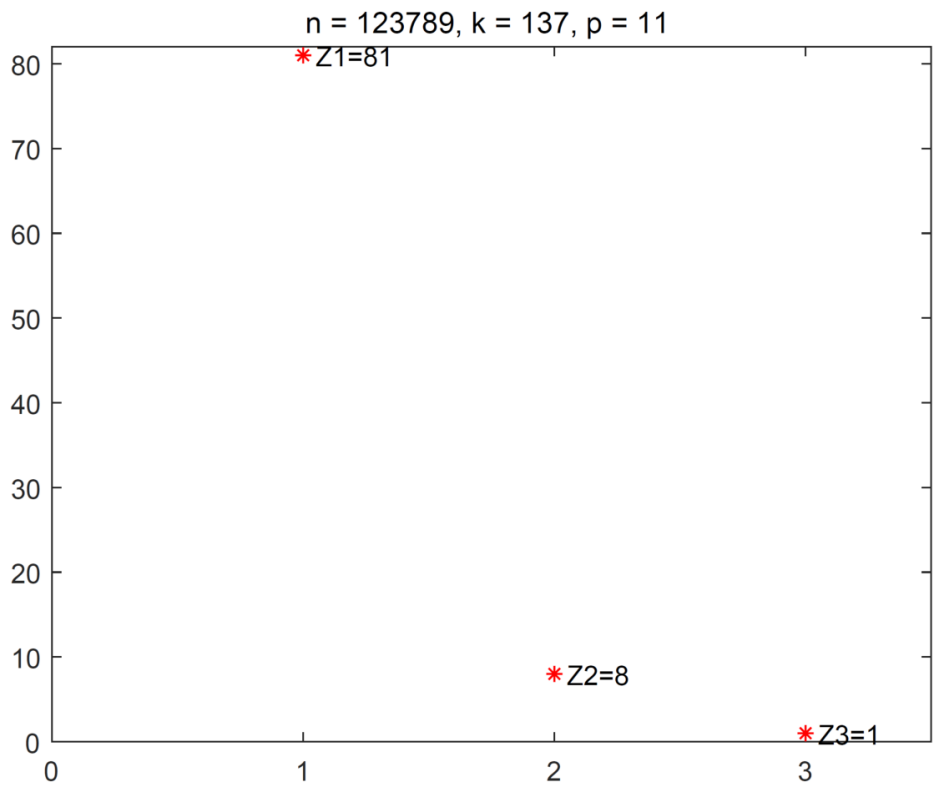

Figure 1. A set of $n, k, p$, which satisfies the Hypothesis. 


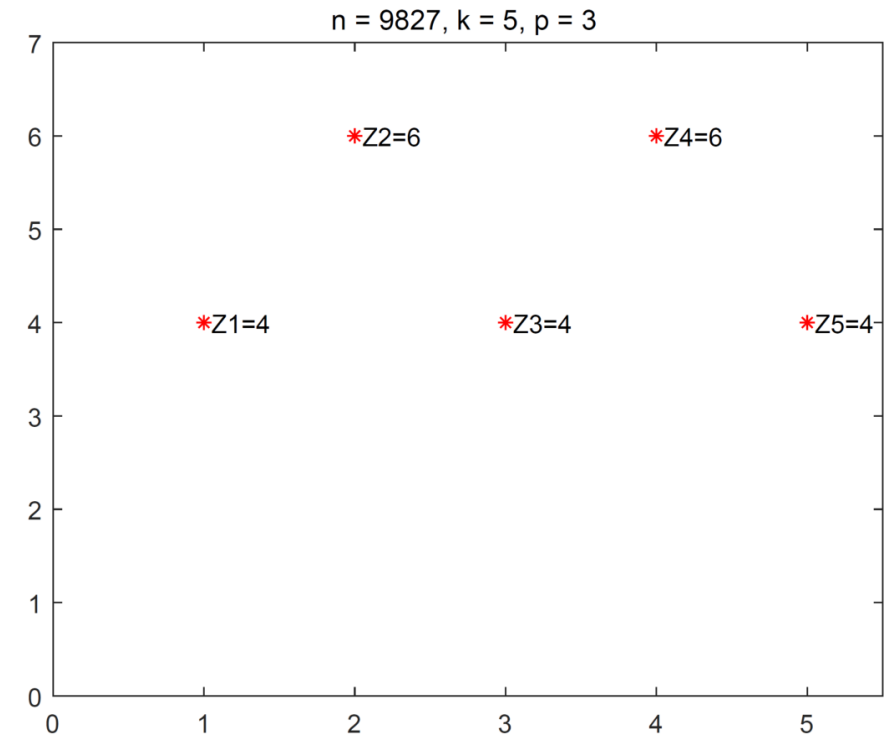

(i) Doesn't satisfy (b) in the Hypothesis.

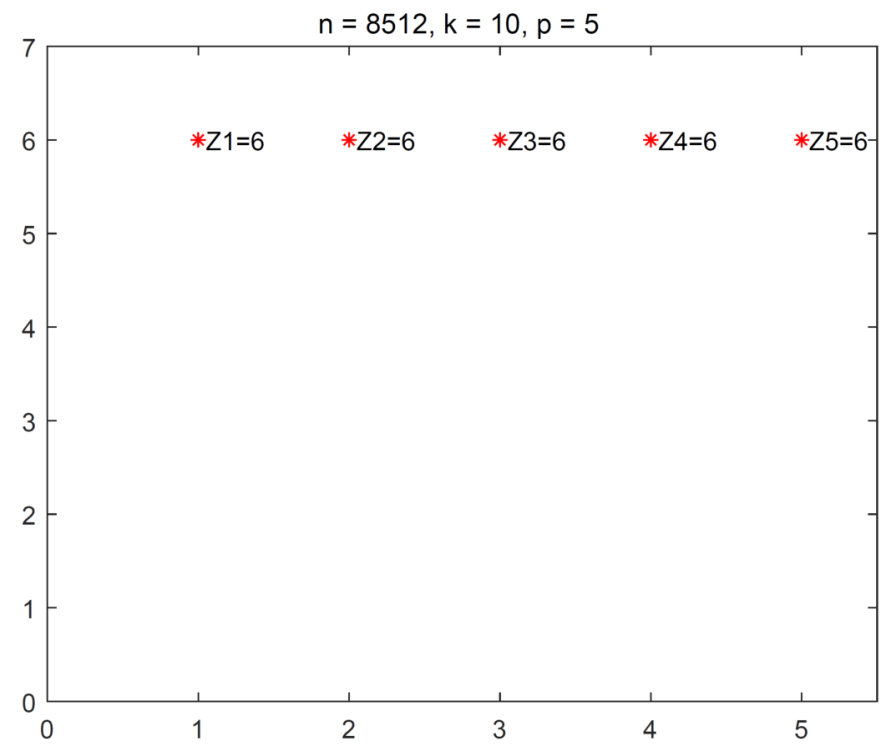

(ii) Doesn't satisfy (c) in the Hypothesis.

Figure 2. Two sets of $n, k, p$, which satisfy the requirement of Theorem 2 . 


\section{Acknowledgements}

This article is supported by Classroom Teaching Innovation Project and Excellent Online Open Course Construction Project of Hangzhou Normal University.

\section{References}

[1] Tianxin Cai, The Book of Numbers, Higher Education Press, 2015, 46-47.

[2] Keith R. Matthews, The generalized $3 x+1$ mapping, Preprint 23pp., dated Oct. 31, 2005.

[3] J. C. Lagarias, The $3 x+1$ problem: An annotated bibliography, II (2000-2009), Mathematics 26(1) (2006), 189-228.

[4] Marc Chamberland, Averaging structure in the $3 x+1$ problem, Journal of Number Theory 148 (2015), 384-397.

DOI: https://doi.org/10.1016/j.jnt.2014.09.024

[5] Aristides V. Doumas and Vassilis G. Papanicolaou, A randomized version of the Collatz $3 x+1$ problem, Statistics \& Probability Letters 109 (2016), 39-44.

DOI: https://doi.org/10.1016/j.spl.2015.10.017

[6] Dora M. Ballesteros, Jimmy Peña and Diego Renza, A novel image encryption scheme based on Collatz conjecture, Entropy 20(12) (2018); Article 901.

DOI: https://doi.org/10.3390/e20120901

[7] Steffen Kionke, A geometric approach to divergent points of higher dimensional Collatz mappings, Monatshefte für Mathematik 182(4) (2017), 851-863.

DOI: https://doi.org/10.1007/s00605-016-0947-4

[8] Ana Caraiani, Multiplicative semigroups related to the $3 x+1$ problem, Advances in Applied Mathematics 45(3) (2010), 373-389.

DOI: https://doi.org/10.1016/j.aam.2010.01.009 